\title{
Predicting product life cycle using fuzzy neural network
}

\author{
Ali Mohammadi ${ }^{\mathrm{a}}$, Aazam Tavakoli ${ }^{\mathrm{b}}$ and Abolghasem Ebrahimi ${ }^{\mathrm{c}^{*}}$
}

${ }^{a}$ Associate Professor, Management Department, Shiraz University, Iran

${ }^{b}$ MA in industrial Management, Management Department, Shiraz University, Iran

${ }^{c}$ Assistance Professor, Management Department, Shiraz University, Iran

\section{H R O N I C L E}

Article history:

Received January 20, 2014

Accepted 5 July 2014

Available online

August 142014

Keywords:

Product Life Cycle (PLC)

Adaptive Network-Based

Fuzzy Inference System (ANFIS)

Neural network

\section{A B S T R A C T}

\begin{abstract}
One of the most important tasks of science in different fields is to find the relationships among various phenomena in order to predict future. Production and service organizations are not exceptions and they should predict future to survive. Predicting the life cycle of the organization's products is one of the most important prediction cases in an organization. Predicting the product life cycle provides an opportunity to identify the product position and help to get a better insight about competitors. This paper deals with the predictability of the product life cycle with Adaptive Network-Based Fuzzy Inference System (ANFIS). The Population of this study was Pegah Fars products and the sample was this company's cheese products. In this regard, this paper attempts to model and predict the product life cycle of cheese products in Pegah Fars Company. In this due, a designed questionnaire was distributed among some experts, distributors and retailers and seven independent variables were selected. In this survey, ANFIS sales forecasting technique was employed and MATLAB software was used for data analysis. The results confirmed ANFIS as a good method to predict the product life cycle.
\end{abstract}

\section{Introduction}

Life cycle theory has been used since the 1970 s to describe the behavior of a product or service from design to obsolescence. The typical pattern of a product is represented by a curve divided into four distinct phases: introduction, growth, maturity, and decline (Rink et al., 1999). After the product is introduced, sales begin growing slowly, until a critical mass of consumer awareness is reached, and then sales grow, rapidly. Eventually, the rapid growth cools down, and the product enters a sustained period of slow growth, or level sales. Eventually, sales will decrease, at first slowly, then more rapidly. Once sales fall below some threshold level, the product will eventually be terminated, and sales will drop to zero (Gallo, 1992). The time length of any stage and the shape of the overall product life cycle (PLC) may vary for various products and industries (Rink et al., 1999). Recent

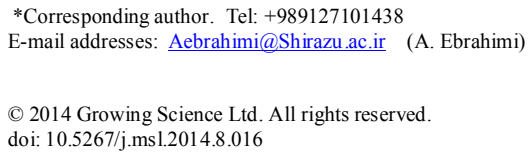


research in the area has concentrated on its use in decision making in areas ranging from those as broad as overall strategy to those as narrow as equipment replacement (Ryan et al., 1996).

The PLC can be a valid framework for evaluating competitive dynamics, the organizational response in terms of marketing mix variables and the sales force compensation strategy based on sales efforts. The compensation system should be designed to reinforce the overall strategy of the organization and should be guided by the stages in the PLC (Madhani, 2011). The recognition of patterns is the basis of all sciences (Watanabe, 1985). In fact, pattern recognition systems are systems that automatically identify objects based on their measured properties or features derived from these properties. With this viewpoint, a neural network also is a pattern recognition system (Lia et al., 2002). Recently, artificial neural networks (ANNs) have been accepted as a potentially useful tool for modeling complex non-linear systems and widely used for prediction (Ham \& Kostanic, 2001).

Since fuzzy set theory was suggested by Zadeh (1965), pattern recognition problems have been intensively studied with fuzzy set (Bezdek, 1981). The revolutionary significance of fuzzy set theory is to provide a mathematical method for describing intuitive knowledge of humans. In principle, a mathematical model constructed in accordance with the classical theory must be interpreted in natural language that could be understood intuitively. In contrast to classical methodology, a fuzzy approach to modeling begins with a practical interpretation of concepts, and then generates intuitive logical relations between concepts and constructs of a model. A model constructed in accordance with the fuzzy theory, therefore, is certainly interpretable (Turksen, 1991).

In recent years, a great deal of attention has been directed towards applying the fusion of fuzzy logic and neural networks to develop intelligent systems. This is because the two technologies are strongly complementary to each other (Lia et al., 2002).

\section{The methodology of ANFIS}

Since Zadeh (1965) proposed the fuzzy logic theorem to describe complicated systems, it has become popular and successfully used for many problems. The main problem with fuzzy logic is that there was no systematic procedure for the design of a fuzzy controller. On the other hand, a neural network has the ability to learn from the environment (input-output pairs), self-organize its structure, and adapt to it in an interactive manner. For this reason, we propose the use of the adaptive neuro-fuzzy inference system (ANFIS) methodology (Jang, 1993) to self-organize network structure and to adapt parameters of the fuzzy system for predicting the product life cycle.

\subsection{Architecture and algorithm}

The ANFIS is a multilayer feed forward network, which uses neural network learning algorithms and fuzzy reasoning to map an input space to an output space. With the ability to combine the verbal power of a fuzzy system with the numeric power of a neural system adaptive network, ANFIS has been shown to be powerful in modeling numerous processes, such as motor fault detection and diagnosis (Altug, Chow \& Trussell , 1999), power systems dynamic load (Djukanovic et al., 1997) ,(Oonsivilai \& El-Hawary, 1999), wind speed (Sfetsos. 2000), forecasting system for the demand of teaching human resources (Liao, $\mathrm{Su} \& \mathrm{Wu}, 2001$ ), and real time reservoir operation (Chang et al., 2005). ANFIS possesses good capability of learning, constructing, expensing, and classifying different things. It has the advantage of allowing the extraction of fuzzy rules from numerical data or expert knowledge and adaptively constructs a rule base. Furthermore, it can tune the complicated conversion of human intelligence to fuzzy systems. The main drawback of the ANFIS forecasting model is the time requested for training structure and determining parameters, which take much time. For simplicity, we assume the fuzzy inference system under consideration has two inputs, $\mathrm{x}$ and $\mathrm{y}$, 
and one output, z. For a first-order Sugeno fuzzy model (Takagi \& Sugeno, 1985), a typical rule set with two fuzzy if-then rules can be expressed as

Rule 1: If $\mathrm{x}$ is $A_{1}$ and $\mathrm{y}$ is $B_{1}$ then $\mathrm{Z} 1=\mathrm{p}_{1} * x+\mathrm{q}_{1} * \mathrm{y}+\mathrm{r} 1$

Rule 2: If $\mathrm{x}$ is $A_{2}$ and $\mathrm{y}$ is $B_{2}$ then $\mathrm{Z} 2=\mathrm{p}_{2} * x+\mathrm{q}_{2} * \mathrm{y}+\mathrm{r} 2$

where pi, qi and ri ( $i=1$ or 2$)$ are linear parameters in the then-part (consequent part) of the firstorder Sugeno fuzzy model. The architecture of ANFIS consists of five layers (Fig. 1), and a brief introduction of the model is as follows.

Layer 1: input nodes. Each node of this layer generates membership grades to which they belong and each of the appropriate fuzzy sets using membership functions.

$$
O_{1, i}=\mu_{A_{i}}(x) \quad \text { for } i=1,2 \quad O_{1, i}=\mu_{B_{i-2}}(y) \quad \text { for } i=3,4
$$

where $x, y$ are the crisp inputs to node $i$, and $A_{i}, B_{i}$ (small, large, etc.) are the linguistic labels characterized by appropriate membership functions $\mu_{A_{i}}, \mu_{B_{i}}$ respectively. Due to smoothness and concise notation, the Gaussian and bell-shaped membership functions are increasingly popular for specifying fuzzy sets. The bell-shaped membership functions have one more parameter than the Gaussian membership functions, so a non-fuzzy set can be approached when the free parameter is tuned.

$\mu_{A_{i}}=\frac{1}{1+\left|\frac{x-c_{i}}{a_{i}}\right|^{2 b_{i}}} \quad \mu_{B_{i-2}}=\frac{1}{1+\left|\frac{y-c_{i}}{a_{i}}\right|^{2 b_{i}}}$

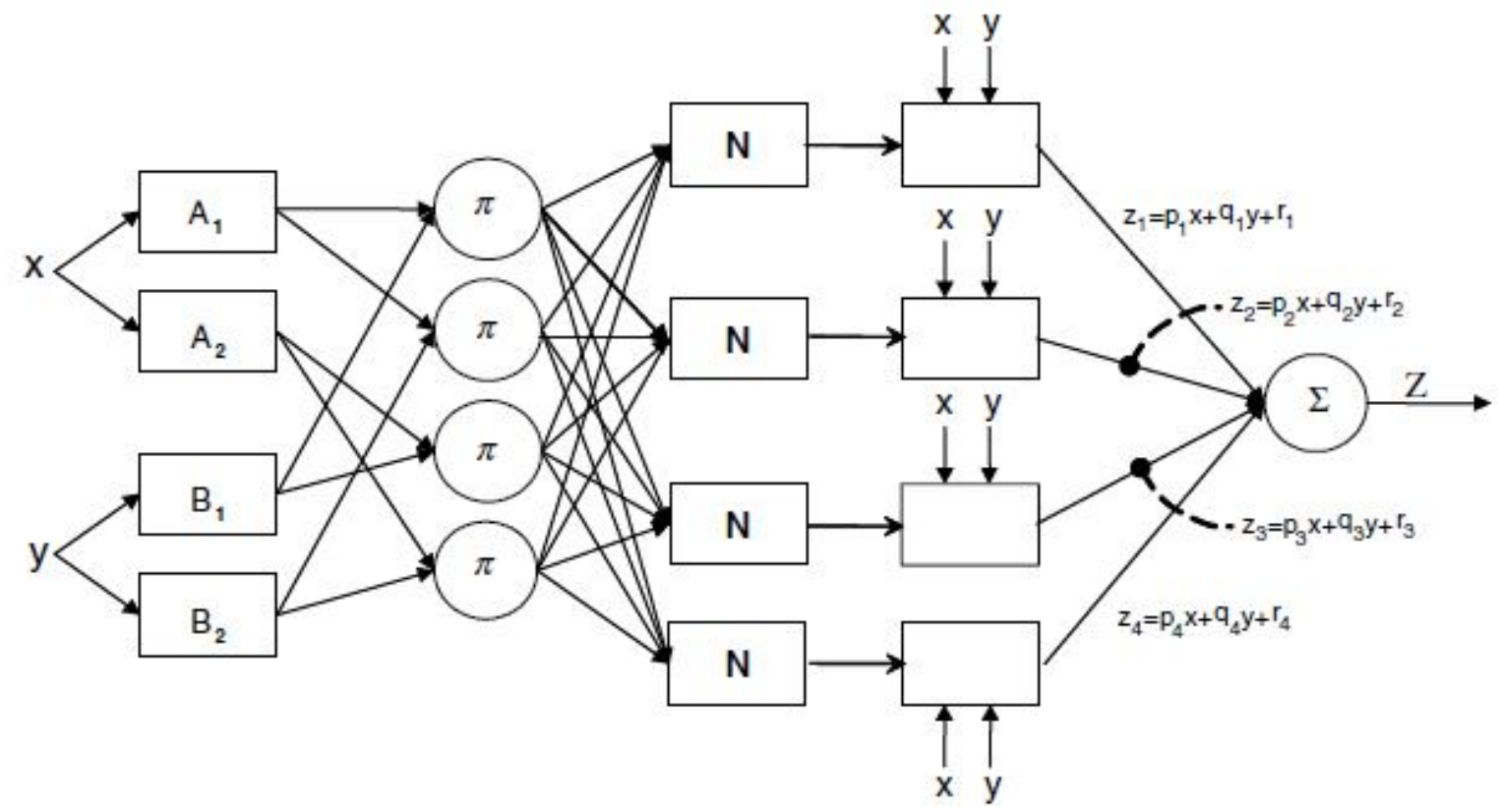

Fig. 1. ANFIS architecture for two-input Sugeno fuzzy model with four rules

where $\left\{a_{i}, b_{i}, c_{i}\right\}$ is the parameter set of the membership functions in the premise part of fuzzy, ifthen rules that change the shapes of the membership function. Parameters in this layer are referred to as the premise parameters.

Layer 2: rule nodes. In the second layer, the AND operator is applied to obtain one output that represents the result of the antecedent for that rule, i.e., firing strength. Firing strength means the 
degrees to which the antecedent part of a fuzzy rule is satisfied and it shapes the output function for the rule. Hence, the outputs $O_{2, k}$ of this layer are the products of the corresponding degrees from Layer 1

$O_{2, k}=w_{k}=\mu_{A_{i}}(x) \times \mu_{B_{j}}(y) \quad k=1, \ldots, 4 ; i=1,2 ; j=1,2$

Layer 3: Average nodes: In the third layer, the main objective is to calculate the ratio of each $i^{\text {th }}$ rules firing strength to the sum of all rules_ firing strength. Consequently, $\bar{w}_{i}$ is taken as the normalized firing strength

$O_{3, i}=\bar{w}_{i}=\frac{w_{i}}{\sum_{k=1}^{4} w_{k}} \quad i=1, \ldots, 4$

Layer 4: consequent nodes. The node function of the fourth layer computes the contribution of each $i^{\text {th }}$ rules toward the total output and the function defined as

$O_{4, i}=\bar{w}_{i} f_{i}=w_{i}\left(p_{i} x+q_{i} y+r_{i}\right), \quad i=1, \ldots, 4$

where $w_{i}$ is the $i^{\text {th }}$ nodes output from the previous layer, as for $\left\{p_{i}, q_{i}, r_{i}\right\}$, they are the coefficients of this linear combination and also the parameter set in the consequent part of the Sugeno fuzzy model.

Layer 5: output nodes.: The single node computes the overall output by summing all the incoming signals. Accordingly, the defuzzification process transforms each rules fuzzy results into a crisp output in this layer

$O_{5,1}=\sum_{i=1}^{4} \bar{w}_{i} f_{i}=\frac{\sum_{i=1}^{4} w_{i} f_{i}}{\sum_{i=1}^{4} w_{i}}$

This network is trained based on supervised learning. So our goal is to train adaptive networks to be able to estimate unknown functions given by training data and then determine the precise value of the above parameters. The distinguishing characteristic of the approach is that ANFIS applies a hybridlearning algorithm, the gradient descent method and the least-squares method, to update parameters. The gradient descent method is employed to tune premise non-linear parameters $\left(\left\{a_{i}, b_{i}, c_{i}\right\}\right)$, while the least-squares method is used to identify consequent linear parameters $\left(\left\{p_{i}, q_{i}, r_{i}\right\}\right)$. As seen in Fig. 1 , the circular nodes are fixed (i.e., not adaptive) nodes without parameter variables, and the square nodes have parameter variables (the parameters are changed during training).

The task of the learning procedure has two steps: In the first step, the least square method identifies the consequent parameters, while the antecedent parameters (membership functions) are assumed to be fixed for the current cycle through the training set. Then, the error signals propagate backward. Gradient descent method is used to update the premise parameters, through minimizing the overall quadratic cost function, while the consequent parameters remain fixed. The detailed algorithm and mathematical background of the hybrid-learning algorithm can be found in.

\section{Choice of the input factors}

To do this prediction, it needs to describe which factors are relevant to the product life cycle. To obtain the variables influencing the PLC, the study has used questionnaire methods. The proposed case study of this paper consists of different parts including production, quality, commercial and technical. In our survey, 8 questionnaires were given to distributors, 7 questionnaires were distributed among some of retailers and 4 questionnaires were distributed and sales representatives. In the questionnaires, they were asked to name 20 factors influencing the PLC. By classifying these factors 
in those same categories, eventually, 7 factors were identified, which include merit product, price, advertising, competitors, distribution, iteration sale month and product type.

The merit product variable is divided into 7 separate variables with sum scores of these variables, the variable product merit is achieved. 7 variables that determine the product merit are quality of raw material, texture quality, taste quality, packaging quality, beauty of packaging, weight and being new. Then these factors are rated according to the desired product. There are seven factors being used for the inputs to the artificial ANFIS proposed in this paper and the output is product life cycle.

\section{Case study}

\section{Establishment of fuzzy rule bases by the subtractive fuzzy clustering}

In this survey, Pegah Fars products were selected as population and cheese product were selected as samples. In this regard, this paper attempts to model and to predict product life cycle cheese product in Pegah Fars Company. The ANFIS models are built to create the fuzzy inference system and then to estimate the PLC based on given input-output patterns. As mentioned above, there are 7 input variables. The subtractive fuzzy clustering can automatically determine the number of clusters. It assumes each data point is a potential cluster center and calculates a measure of the likelihood that each data point would define the cluster center, based on the density of surrounding data points. First, each data point is considered as a potential cluster center instead of grid point. The density measure of each data point $x_{i}$ is defined as

$D_{i}=\sum_{j=1}^{n} \exp \left(-\frac{\left\|x_{i}-x_{j}\right\|^{2}}{\left(r_{a} / 2\right)^{2}}\right)$

where the positive constant $r_{a}$ is the radius defining a neighborhood of a cluster center. After the density measure of each data point is selected, the point with the highest density $D_{c 1}$ as the first cluster center $x_{c 1}$ is selected. In order to avoid neighborhood points of the first cluster center being selected as the second center, the density measure of each data point $x_{i}$ is revised as

$D_{i}=D_{i}-D_{c 1} \exp \left(-\frac{\left\|x_{i}-x_{c 1}\right\|^{2}}{\left(r_{b} / 2\right)^{2}}\right)$

The recommended value of setting $r_{b}$ is equal to $1.5 r_{a}$. The modification of the density measure in the remaining point set is applied in each step. The process of determining the cluster center and its corresponding density are repeated until some stop conditions are met. After several numbers of clustering are evaluated, each forecasting model adopts appropriate number of rules. It appears that the subtractive fuzzy clustering does significantly reduce the number of rules, where all forecasting models only need a few number of rules. That means the vast and complex input-output patterns ( 7 input variables and one output) could be efficiently clustered into a few of rules, and those rules could make an accurate forecasting model.

\section{Performance measures}

To obtain the better performance of learning, it is necessary to choose the proper parameters values and use them to train the ANFIS predictive model in this paper. There are two indices used to measure the training and test performance. They are root mean squared error (RMSE) and correlation coefficient (R), 


$$
\begin{aligned}
& R M S E=\sqrt{\frac{1}{n} \sum_{i=1}^{n}\left(q_{o}(t)-q_{p}(t)\right)^{2}}, \\
& R^{2}=\frac{\sum_{i=1}^{n}\left(q_{o}(t)-\bar{q}_{o}(t)\right)\left(q_{p}(t)-\bar{q}_{p}(t)\right)}{\left(\sum_{i=1}^{n}\left(q_{o}(t)-\bar{q}_{o}(t)\right)^{2}\right)^{0 / 5}\left(\sum_{i=1}^{n}\left(q_{p}(t)-\bar{q}_{p}(t)\right)^{2}\right)^{0 / 5}},
\end{aligned}
$$

where $n$ is the number of training data in an epoch, $q_{0}$ and $q_{p}$ are the desired output value and ANFIS's output value for the ith training datum of the epoch, respectively.

\section{Results and discussion}

Subclustering method of fuzzy inference systems tried to generate the fuzzy rule base sets. A number of 60 epochs for training was applied to obtain the minimum root mean square error (RMSE) and maximum square of correlation coefficient $\left(R^{2}\right)$ that the perfect agreement is achieved when RMSE and $R^{2}$ are equal to 0.0 and 1.0 respectively. The data set was divided randomly into three subsets as $70 \%$ for training, $15 \%$ for testing and $15 \%$ for checking purposes. More data is used in the training phase because ANFIS is more adapted nonlinear functional dependency between input and output variables. Generally, fuzzy rules were described by an expert. Instead of consulting an expert, the rules were automatically generated in this study. The optimum ANFIS structures were obtained by trial and error of grid partition fuzzy inference system and the lowest RMSE and highest $R^{2}$ values were obtained with subclustering system.

\section{Table 1}

\begin{tabular}{|c|c|c|c|c|c|c|c|}
\hline \multicolumn{8}{|c|}{ Adaptive Network-Based Fuzzy Inference System (ANFIS) } \\
\hline \multicolumn{8}{|c|}{ Subtractive fuzzy clustering } \\
\hline Month & Stage & RMSE & $\mathrm{R}^{2}$ & Month & Stage & RMSE & $\mathrm{R}^{2}$ \\
\hline \multirow{3}{*}{ April } & train & 18.83 & 0.99 & \multirow{3}{*}{ October } & train & 29.88 & 0.99 \\
\hline & test & 1106.16 & 0.34 & & test & 2799.1 & 0.3 \\
\hline & check & 2320.75 & 0.66 & & check & 3630.88 & 0.074 \\
\hline \multirow{3}{*}{ May } & train & 43.41 & 0.99 & \multirow{3}{*}{ November } & train & 0.00051 & 0.99 \\
\hline & test & 2627.87 & 0.69 & & test & 2503.69 & 0.39 \\
\hline & check & 2827.65 & 0.45 & & check & 1595.69 & 0.043 \\
\hline \multirow{3}{*}{ June } & train & 0.001 & 0.99 & \multirow{3}{*}{ December } & train & 0.599 & 0.99 \\
\hline & test & 916.5 & 0.84 & & test & 1975.36 & 0.63 \\
\hline & check & 4353.51 & 0.12 & & check & 2713.6 & 0.4 \\
\hline \multirow{3}{*}{ July } & train & 0.034 & 0.99 & \multirow{3}{*}{ January } & train & 0.0094 & 0.99 \\
\hline & test & 1805.3 & 0.66 & & test & 2170.15 & 0.64 \\
\hline & check & 3484.2 & 0.3 & & check & 2300.69 & 0.45 \\
\hline \multirow{3}{*}{ August } & train & 0.051 & 0.99 & \multirow{3}{*}{ February } & train & 0.965 & 0.99 \\
\hline & test & 2010.91 & 0.53 & & test & 1738.98 & 0.56 \\
\hline & check & 2192.24 & 0.47 & & check & 2896.97 & 0.34 \\
\hline \multirow{3}{*}{ September } & train & 0.248 & 0.99 & \multirow{3}{*}{ March } & train & 0.044 & 0.99 \\
\hline & test & 4158.62 & 0.57 & & test & 2621.09 & 0.23 \\
\hline & check & 3155.49 & 0.19 & & check & 1906.08 & 0.7 \\
\hline
\end{tabular}

Predicting using fuzzy neural network

Predicting plc for chess A:

At this stage, examine capability of model using data of product A. First, we need determine the inputs to this product. The following summarizes the necessary information for product A: 


\begin{tabular}{ccccccc}
\hline Product & Product & Price & Advertising & Competitors & Distribution & Iteration sale \\
\hline 8 & 40 & 20 & 80 & 30 & 40 & 1 \\
\hline
\end{tabular}

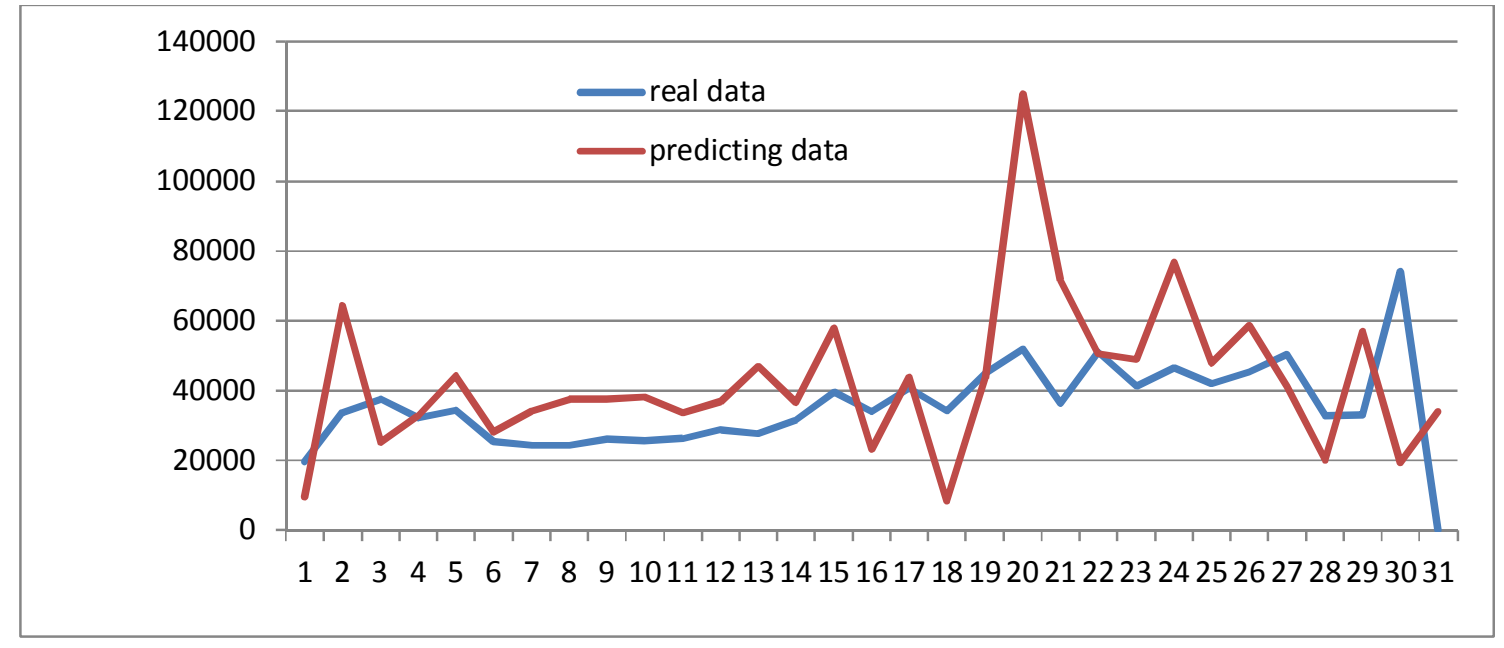

Fig. 2. Comparison of the actual life cycle and predicted life cycle

\section{Conclusions}

In this paper, an Adaptive Network-Based Fuzzy Inference System (ANFIS) predictive model was proposed. The model can be applied for predicting product life cycle and recommendations to managers and marketers. After learning the training data, the trained ANFIS predictive model can infer the unseen test data with accuracy. The results of training and test performance have shown that the ANFIS predictive model was a good forecasting method in complex and various environments, even if the underlying relationship between inputs and outputs were nonlinear.

\section{Acknowledgement}

The authors would like to thank the anonymous referees for constructive comments on earlier version of this paper.

\section{References}

Altug, S., Chen, M. Y., \& Trussell, H. J. (1999). Fuzzy inference systems implemented on neural architectures for motor fault detection and diagnosis. Industrial Electronics, IEEE Transactions on, 46(6), 1069-1079.

Bezdek, J. C. (1981). Pattern recognition with fuzzy objective function algorithms. Kluwer Academic Publishers.

Chang, L. C., \& Chang, F. J. (2001). Intelligent control for modelling of real-time reservoir operation. Hydrological Processes, 15(9), 1621-1634.

Djukanovic, M. B., Calovic, M. S., Vesovic, B. V., \& Sobajic, D. J. (1997). Neuro-fuzzy controller of low head hydropower plants using adaptive-network based fuzzy inference system. Energy Conversion, IEEE Transactions on,12(4), 375-381.

Gallo, A. E. (1992). Record number of new products in 1991. Food Review,15(3), 19-21.

Ham, F. M., \& Kostanic, I. (2000). Principles of neurocomputing for science and engineering. McGraw-Hill Higher Education.

Jang, J. S. (1993). ANFIS: adaptive-network-based fuzzy inference system.Systems, Man and Cybernetics, IEEE Transactions on, 23(3), 665-685. 
Liao, H. P., Su, J. P., \& Wu, H. M. (2001). An Application of ANFIS to Modeling of a Forecasting System for the Demand of Teacher Human Resources (Article written in Chinese). Journal of Education and Psychology,24(1), 1-17.

Li, R. P., Mukaidono, M., \& Turksen, I. B. (2002). A fuzzy neural network for pattern classification and feature selection. Fuzzy Sets and Systems, 130(1), 101-108.

Madhani, P. M. (2011). Restructuring fixed and variable pay in sales organizations: a product life cycle approach. Compensation \& Benefits Review,43(4), 245-258.

Oonsivilai, A., \& El-Hawary, M. E. (1999, May). Power system dynamic load modeling using adaptive-network-based fuzzy inference system. In Electrical and Computer Engineering, 1999 IEEE Canadian Conference on (Vol. 3, pp. 1217-1222). IEEE.

Ryan, C., \& Riggs, W. E. (1996). Redefining the product life cycle: the five-element product wave. Business Horizons, 39(5), 33-40.

Rink, D. R., Roden, D. M., \& Fox, H. W. (1999). Financial management and planning with the product life cycle concept. Business Horizons, 42(5), 65-72.

Sfetsos, A. (2000). A comparison of various forecasting techniques applied to mean hourly wind speed time series. Renewable energy, 21(1), 23-35.

Turksen, I. B. (1991). Measurement of membership functions and their acquisition. Fuzzy sets and systems, 40(1), 5-38.

Takagi, T., \& Sugeno, M. (1985). Fuzzy identification of systems and its applications to modeling and control. Systems, Man and Cybernetics, IEEE Transactions on, (1), 116-132.

Watanabe, S. (1985). Pattern recognition: human and mechanical. John Wiley \& Sons, Inc.

Zadeh, L.A. (1965). Fuzzy Sets. Information and Control, 8, 338-353. 